\title{
Integral Transforms of Square Integrable Functionals on Yeh- Wiener Space
}

\section{Byoung SoO KIM}

School of Liberal Arts, Seoul National University of Technology, Seoul 139-743, Korea

e-mail : mathkbs@snut.ac.kr

ABSTRACT. We give a necessary and sufficient condition that a square integrable functional $F(x)$ on Yeh-Wiener space has an integral transform $\hat{\mathcal{F}}_{\alpha, \beta} F(x)$ which is also square integrable. This extends the result by Kim and Skoug for functional $F(x)$ in $L_{2}\left(C_{0}[0, T]\right)$.

\section{Introduction and definitions}

Let $C(Q)$ denote Yeh-Wiener space; that is the space of all real-valued continuous functions $x(s, t)$ on $Q=[0, S] \times[0, T]$ with $x(s, 0)=x(0, t)=0$ for all $0 \leq s \leq S$ and $0 \leq t \leq T$. Yeh [14] defined a Gaussian measure $m_{Y}$ on $C(Q)$ (later modified in [16]) such that as a stochastic process $\{x(s, t):(s, t) \in Q\}$ has mean $E[x(s, t)]=0$ and covariance $E[x(s, t) x(u, v)]=\min \{s, u\} \min \{t, v\}$.

Let $\mathcal{M}$ denote the class of all Yeh-Wiener measurable subsets of $C(Q)$ and we denote the Yeh-Wiener integral of a Yeh-Wiener integrable functional $F$ by

$$
\int_{C(Q)} F(x) m_{Y}(d x) .
$$

Let $L_{2}(C(Q))$ be the space of all real or complex valued functionals $F$ satisfying

$$
\int_{C(Q)}|F(x)|^{2} m_{Y}(d x)<\infty .
$$

Let $K(Q)$ be the space of complex valued continuous functions defined on $Q$ and satisfying $x(s, 0)=x(0, t)=0$ for all $0 \leq s \leq S$ and $0 \leq t \leq T$. Let $\alpha$ and $\beta$ be nonzero complex numbers. Next we state the definitions of the integral transform $\mathcal{F}_{\alpha, \beta} F$ introduced in [12] and studied in [6],[9],[10] and [11].

Definition 1.1. Let $F$ be a functional defined on $K(Q)$. Then the integral transform $\mathcal{F}_{\alpha, \beta} F$ of $F$ is defined by

$$
\mathcal{F}_{\alpha, \beta} F(y)=\int_{C(Q)} F(\alpha x+\beta y) m_{Y}(d x), \quad y \in K(Q)
$$

Received 24 January 2008; accepted 1 April 2008.

2000 Mathematics Subject Classification: 28C20, 60J65.

Key words and phrases: Yeh-Wiener integral, integral transform, Fourier-Wiener transform, Fourier-Hermite functional. 
if it exists.

Remark 1.2. (1) When $\alpha=1$ and $\beta=i, \mathcal{F}_{\alpha, \beta} F$ is a Yeh-Wiener space version of the Fourier-Wiener transform introduced by Cameron in [2] and used by Cameron and Martin in [3].

(2) When $\alpha=\sqrt{2}$ and $\beta=i, \mathcal{F}_{\alpha, \beta} F$ is a Yeh-Wiener space version of the modified Fourier-Wiener transform introduced by Cameron and Martin in [4].

(3) Equation (1.1) implies that

$$
\mathcal{F}_{\alpha, \beta \beta^{\prime}} F(y)=\mathcal{F}_{\alpha, \beta} F\left(\beta^{\prime} y\right), \quad y \in K(Q)
$$

for any nonzero complex number $\beta^{\prime}$.

(4) For a detailed survey of previous work on integral transform, Fourier-Wiener transform and Fourier-Feynman transform [5], see [13].

Recently Kim and Skoug [11] established a necessary and sufficient condition that a functional $F(x)$ in $L_{2}\left(C_{0}[0, T]\right)$ has an integral transform $\mathcal{F}_{\alpha, \beta} F(x)$ which also belong to $L_{2}\left(C_{0}[0, T]\right)$. In this paper we extend this result for square integrable functionals on Yeh-Wiener space, that is, we give a necessary and sufficient condition that a functional $F(x)$ in $L_{2}(C(Q))$ has an integral transform $\hat{\mathcal{F}}_{\alpha, \beta} F(x)$, which will be defined in Section 3, also belonging to $L_{2}(C(Q))$.

Now we introduce a concept of the function of bounded variation of two variables. The concept of bounded variation for a function of two variables is surprisingly complex. In this paper we will use the definition used by Hardy and Krause $[1],[8]$ which we now review.

Let $R=[a, b] \times[c, d]$ and let $P$ be a partition of $R$ given by

$$
a=s_{0}<s_{1}<\cdots<s_{n}=b, \quad c=t_{0}<t_{1}<\cdots<t_{m}=d .
$$

A function $f(s, t)$ is said to be of bounded variation on $R$ in the sense of Hardy and Krause provided the following three conditions hold.

(1) There is a constant $B$ such that

$$
\sum_{i=1}^{n} \sum_{j=1}^{m}\left|f\left(s_{i}, t_{j}\right)-f\left(s_{i}, t_{j-1}\right)-f\left(s_{i-1}, t_{j}\right)+f\left(s_{i-1}, t_{j-1}\right)\right| \leq B
$$

for all partitions $P$.

(2) For each $t \in[c, d], f(\cdot, t)$ is a function of bounded variation on $[a, b]$.

(3) For each $s \in[a, b], f(s, \cdot)$ is a function of bounded variation on $[c, d]$.

The total variation $\operatorname{Var}(f, R)$ of $f$ over $R$ is defined to be the supremum of the sums in (1.3) over all partitions $P$ of $R$. $\operatorname{Var}(f(\cdot, t),[a, b])$ and $\operatorname{Var}(f(s, \cdot),[c, d])$ will denote the total variation of $f(\cdot, t)$ on $[a, b]$ and $f(s, \cdot)$ on $[c, d]$, respectively, as functions of single variable.

The definition of bounded variation by Hardy and Krause has the important property that if $g$ is continuous on $R$ and $f$ is of bounded variation on $R$ then the 
Riemann-Stieltjes integrals $\int_{R} g(s, t) d f(s, t)$ and $\int_{R} f(s, t) d g(s, t)$ both exist and satisfy an integration by parts formula [7].

Let $\left\{\theta_{1}, \theta_{2}, \cdots, \theta_{n}\right\}$ be an orthonormal set of real-valued functions in $L_{2}(C(Q))$. Furthermore assume that each $\theta_{j}$ is of bounded variation in the sense of Hardy and Krause on $Q$. Then for each $y \in K(Q)$ and $j=1,2, \cdots$, the RiemannStieltjes integral $\left\langle\theta_{j}, y\right\rangle \equiv \int_{Q} \theta_{j}(s, t) d y(s, t)$ exists. We finish this section by introducing a well-known Yeh-Wiener integration formula for functionals $f(\langle\vec{\theta}, x\rangle) \equiv$ $f\left(\left\langle\theta_{1}, x\right\rangle, \cdots,\left\langle\theta_{n}, x\right\rangle\right)$ :

$$
\int_{C(Q)} f(\langle\vec{\theta}, x\rangle) m_{Y}(d x)=(2 \pi)^{-n / 2} \int_{\mathbb{R}^{n}} f(\vec{u}) \exp \left\{-\frac{1}{2}\|\vec{u}\|^{2}\right\} d \vec{u},
$$

where $\|\vec{u}\|^{2}=\sum_{j=1}^{n} u_{j}^{2}$ and $d \vec{u}=d u_{1} \cdots d u_{n}$.

\section{Integral transforms of the Fourier-Hermite functionals}

For $n=0,1,2, \cdots$, let $H_{n}(u)$ denote the Hermite polynomial

$$
H_{n}(u)=(-1)^{n}(n !)^{-1 / 2} e^{u^{2} / 2} \frac{d^{n}}{d u^{n}}\left(e^{-u^{2} / 2}\right) .
$$

Then, as is well known, the set

$$
\left\{(2 \pi)^{-1 / 4} H_{n}(u) e^{-u^{2} / 4}: n=0,1,2, \cdots\right\}
$$

is a complete orthonormal $(\mathrm{CON})$ set on $\mathbb{R}$.

Let $\left\{\theta_{p}(s, t): p=1,2, \cdots\right\}$ be a CON set of functions of bounded variation on $Q$. Define

$$
\Phi_{n, p}(y)=H_{n}\left(\left\langle\theta_{p}, y\right\rangle\right), \quad n=0,1,2, \cdots, p=1,2, \cdots,
$$

and

$$
\Psi_{n_{1}, \cdots, n_{p}}(y)=\Psi_{n_{1}, \cdots, n_{p}, 0, \cdots, 0}(y)=\Phi_{n_{1}, 1}(y) \cdots \Phi_{n_{p}, p}(y)
$$

for $y \in K(Q)$. The functionals in (2.2) are called the Fourier-Hermite functionals on Yeh-Wiener space.

In [15], Yeh showed that the Fourier-Hermite functionals form a CON set in $L_{2}(C(Q))$. That is to say that every functional $F(x)$ in $L_{2}(C(Q))$ has a FourierHermite development which converges in the $L_{2}(C(Q))$ sense to $F(x)$; namely that

$$
F(x)=\underset{N \rightarrow \infty}{\lim } \sum_{n_{1}, \cdots, n_{N}=0}^{N} A_{n_{1}, \cdots, n_{N}}^{F} \Psi_{n_{1}, \cdots, n_{N}}(x),
$$

where $A_{n_{1}, \cdots, n_{N}}^{F}$ is the Fourier-Hermite coefficient

$$
A_{n_{1}, \cdots, n_{N}}^{F}=\int_{C(Q)} F(x) \Psi_{n_{1}, \cdots, n_{N}}(x) m_{Y}(d x) .
$$


Throughout this paper, in order to insure that various integrals exist, we will assume that $\beta=a+b i$ is a nonzero complex number satisfying the inequality

$$
\operatorname{Re}\left(1-\beta^{2}\right)=1+b^{2}-a^{2}>0 .
$$

Note that $\operatorname{Re}\left(1-\beta^{2}\right)=1+b^{2}-a^{2}>0$ if and only if the point $(a, b) \in \mathbb{R}^{2}$ lies in the open region, determined by the hyperbola $a^{2}-b^{2}=1$, containing the $b$-axis. Hence for all $|\beta| \leq 1, \beta \neq \pm 1, \operatorname{Re}\left(1-\beta^{2}\right)>0$. Next we define

$$
\alpha \equiv \sqrt{1-\beta^{2}}, \quad-\pi / 4<\arg (\alpha)<\pi / 4
$$

and note that $\alpha^{2}+\beta^{2}=1$ and $\operatorname{Re}\left(\alpha^{2}\right)=\operatorname{Re}\left(1-\beta^{2}\right)>0$.

The following lemma is introduced in [11] and will be needed to find the integral transform of the Fourier-Hermite functionals on Yeh-Wiener space.

Lemma 2.1. Let $\beta$ be a nonzero complex number satisfying inequality (2.5) and let $\alpha$ be defined by equation (2.6). Let $r$ be a complex number. Then for $n=0,1,2, \cdots$,

$$
\int_{\mathbb{R}} H_{n}(u) \exp \left\{-\frac{1}{2 \alpha^{2}}(u-r \beta)^{2}\right\} d u=\sqrt{2 \pi} \alpha \beta^{n} H_{n}(r) .
$$

Next, using Lemma 2.1, we obtain a formula for the integral transform of the Fourier-Hermite functionals given by equation (2.2).

Theorem 2.2. Let $\alpha$ and $\beta$ be as in Lemma 2.1. Then for each $y \in K(Q)$,

$$
\mathcal{F}_{\alpha, \beta} \Psi_{n_{1}, \cdots, n_{p}}(y)=\beta^{n_{1}+\cdots+n_{p}} \Psi_{n_{1}, \cdots, n_{p}}(y) .
$$

Proof. For $j=1,2, \cdots$, let $r_{j}=\left\langle\theta_{j}, y\right\rangle$ which we know exists for all $y \in K(Q)$ since $\theta_{j}$ is of bounded variation on $Q$. Then for every $y \in K(Q)$, by the Yeh-Wiener integration formula (1.4),

$$
\begin{aligned}
\mathcal{F}_{\alpha, \beta} \Psi_{n_{1}, \cdots, n_{p}}(y) & =\int_{C(Q)} \Psi_{n_{1}, \cdots, n_{p}}(\alpha x+\beta y) m_{Y}(d x) \\
& =\prod_{j=1}^{p}\left[(2 \pi)^{-1 / 2} \int_{\mathbb{R}} H_{n_{j}}\left(\alpha u_{j}+\beta r_{j}\right) e^{-u_{j}^{2} / 2} d u_{j}\right] .
\end{aligned}
$$

Note that for all positive $\alpha$ and all $\beta \in \mathbb{C}$,

$$
\int_{\mathbb{R}} H_{n}(\alpha u+\beta r) e^{-u^{2} / 2} d u=\frac{1}{\alpha} \int_{\mathbb{R}} H_{n}(u) e^{-(u-r \beta)^{2} / 2 \alpha^{2}} d u .
$$

But each side of the above expression is an analytic function of $\alpha$ throughout the region $\left\{\alpha \in \mathbb{C}: \operatorname{Re}\left(\alpha^{2}\right)>0\right\}$. Hence by the uniqueness theorem for analytic functions, the above equality holds for all $\alpha$ with $\operatorname{Re}\left(\alpha^{2}\right)>0$ and all $\beta \in \mathbb{C}$ and so

$$
\mathcal{F}_{\alpha, \beta} \Psi_{n_{1}, \cdots, n_{p}}(y)=\prod_{j=1}^{p}\left[\left(2 \pi \alpha^{2}\right)^{-1 / 2} \int_{\mathbb{R}} H_{n_{j}}\left(u_{j}\right) e^{-\left(u_{j}-r_{j} \beta\right)^{2} / 2 \alpha^{2}} d u_{j}\right] .
$$


Then using Lemma 2.1, we obtain equation (2.8), the desired result.

Our first corollary follows immediately from equation (2.8) and the fact that $\left\|\Psi_{n_{1}, \cdots, n_{p}}\right\|_{2}=1$.

Corollary 2.3. Let $\alpha$ and $\beta$ be as in Lemma 2.1. Then

$$
\left\|\mathcal{F}_{\alpha, \beta} \Psi_{n_{1}, \cdots, n_{p}}\right\|_{2}=|\beta|^{n_{1}+\cdots+n_{p}} .
$$

By (1.2) and Theorem 2.2, we obtain the following corollary.

Corollary 2.4. Let $\alpha$ and $\beta$ be as in Lemma 2.1 and let $\gamma$ be any nonzero complex number. Then for each $y \in K(Q)$,

$$
\mathcal{F}_{\alpha, \gamma} \Psi_{n_{1}, \cdots, n_{p}}(y)=\beta^{n_{1}+\cdots+n_{p}} \Psi_{n_{1}, \cdots, n_{p}}\left(\frac{\gamma y}{\beta}\right) .
$$

\section{Integral transforms of functionals belonging to $L_{2}(C(Q))$}

For $F \in L_{2}(C(Q))$ let $(2.3)$ denote the Fourier-Hermite expression of $F(x)$ with the Fourier-Hermite coefficients $A_{n_{1}, \cdots, n_{N}}^{F}$ given by equation (2.4). For $N=1,2, \cdots$, let

$$
F_{N}(x)=\sum_{n_{1}, \cdots, n_{N}=0}^{N} A_{n_{1}, \cdots, n_{N}}^{F} \Psi_{n_{1}, \cdots, n_{N}}(x) .
$$

Then by Theorem 2.2, we know that for each $N=1,2, \cdots$, the integral transform $\mathcal{F}_{\alpha, \beta} F_{N}$ exists for all $\alpha$ and $\beta$ as in Lemma 2.1, and $\mathcal{F}_{\alpha, \beta} F_{N}$ is an element of $L_{2}(C(Q))$ such that for each $y \in K(Q)$,

$$
\mathcal{F}_{\alpha, \beta} F_{N}(y)=\sum_{n_{1}, \cdots, n_{N}=0}^{N} A_{n_{1}, \cdots, n_{N}}^{F} \beta^{n_{1}+\cdots+n_{N}} \Psi_{n_{1}, \cdots, n_{N}}(y) .
$$

Furthermore,

$$
\left\|\mathcal{F}_{\alpha, \beta} F_{N}\right\|_{2}^{2}=\sum_{n_{1}, \cdots, n_{N}=0}^{N}\left|A_{n_{1}, \cdots, n_{N}}^{F} \beta^{n_{1}+\cdots+n_{N}}\right|^{2} .
$$

Definition 3.1. Let $F \in L_{2}(C(Q))$ be given by (2.3). Then for each pair of nonzero complex numbers $\alpha$ and $\beta$, we define the integral transform $\hat{\mathcal{F}}_{\alpha, \beta} F$ of $F$ to be

$$
\hat{\mathcal{F}}_{\alpha, \beta} F(x)=\underset{N \rightarrow \infty}{\lim . \operatorname{m}} \mathcal{F}_{\alpha, \beta} F_{N}(x), \quad x \in C(Q)
$$

if it exists; that is to say if

$$
\lim _{N \rightarrow \infty} \int_{C(Q)}\left|\hat{\mathcal{F}}_{\alpha, \beta} F(x)-\mathcal{F}_{\alpha, \beta} F_{N}(x)\right|^{2} m_{Y}(d x)=0 .
$$


Suppose that $F$ is a functional defined on $K(Q)$ and has the integral transform $\mathcal{F}_{\alpha, \beta} F(y)$ for $y \in K(Q)$ in the sense of Definition 1.1. Further assume that $F$, as a function of $x \in C(Q)$, belongs to $L_{2}(C(Q))$ and has the integral transform $\hat{\mathcal{F}}_{\alpha, \beta} F$ for $x \in C(Q)$ in the sense of Definition 3.1. The following example shows that the two integral transforms $\mathcal{F}_{\alpha, \beta} F(x)$ and $\hat{\mathcal{F}}_{\alpha, \beta} F(x)$ for $x \in C(Q)$ need not coincide.

Example 3.2. Let $F$ be a functional defined on $K(Q)$ by

$$
F(y)= \begin{cases}0, & \text { if } y \in C(Q) \\ 1, & \text { if } y \in K(Q) \backslash C(Q) .\end{cases}
$$

Then $F$ belongs to $L_{2}(C(Q))$ and for $x \in C(Q)$, we have

$$
\mathcal{F}_{\sqrt{2}, i} F(x)=\int_{C(Q)} F(\sqrt{2} z+i x) m_{Y}(d z)=1 .
$$

On the other hand, for any nonnegative integers $n_{1}, \cdots, n_{N}$,

$$
A_{n_{1}, \cdots, n_{N}}^{F}=\int_{C(Q)} F(x) \Psi_{n_{1}, \cdots, n_{N}}(x) m_{Y}(d x)=0
$$

and so $F_{N}(y)=0$ for $y \in K(Q)$ and for all $N=1,2, \cdots$. Now

$$
\mathcal{F}_{\sqrt{2}, i} F_{N}(x)=\int_{C(Q)} F_{N}(\sqrt{2} z+i x) m_{Y}(d z)=0, \quad x \in C(Q)
$$

and so

$$
\hat{\mathcal{F}}_{\sqrt{2}, i} F(x)=\lim _{N \rightarrow \infty} \operatorname{Fi}_{\sqrt{2}, i} F_{N}(x)=0, \quad x \in C(Q) .
$$

Hence we conclude that

$$
\mathcal{F}_{\sqrt{2}, i} F(x) \neq \hat{\mathcal{F}}_{\sqrt{2}, i} F(x)
$$

for $x \in C(Q)$.

Theorem 3.3. Let $F \in L_{2}(C(Q))$ be given by (2.3). Let $\alpha$ and $\beta$ be nonzero complex numbers and let $c$ be a nonzero real number. Then

$$
\hat{\mathcal{F}}_{\alpha, c \beta} F(x)=\hat{\mathcal{F}}_{\alpha, \beta} F(c x)
$$

for $x \in C(Q)$.

Proof. By (1.2) for each $N=1,2, \cdots$,

$$
\mathcal{F}_{\alpha, c \beta} F_{N}(x)=\mathcal{F}_{\alpha, \beta} F_{N}(c x)
$$

and so

$$
\hat{\mathcal{F}}_{\alpha, c \beta} F(x)=\lim _{N \rightarrow \infty} \mathcal{F}_{\alpha, c \beta} F_{N}(x)=\lim _{N \rightarrow \infty} \mathcal{F}_{\alpha, \beta} F_{N}(c x)=\hat{\mathcal{F}}_{\alpha, \beta} F(c x)
$$


as desired.

The following lemma gives us a relationship between the Fourier-Hermite coefficients of $\hat{\mathcal{F}}_{\alpha, \beta} F$ and $F$.

Lemma 3.4. Let $F \in L_{2}(C(Q))$ be given by (2.3) with Fourier-Hermite coefficients given by (2.4). Let $\alpha$ and $\beta$ be as in Lemma 2.1 and assume that $\hat{\mathcal{F}}_{\alpha, \beta} F$ exists and is in $L_{2}(C(Q))$. Then

$$
A_{n_{1}, \cdots, n_{N}}^{\hat{\mathcal{F}}_{\alpha, \beta} F}=A_{n_{1}, \cdots, n_{N}}^{F} \beta^{n_{1}+\cdots+n_{N}}
$$

for each $N=1,2, \cdots$.

Proof. Fix $N=1,2, \cdots$. For any given $\epsilon>0$, take a natural number $M$ satisfying $\left\|\hat{\mathcal{F}}_{\alpha, \beta} F-\mathcal{F}_{\alpha, \beta} F_{M}\right\|_{2}<\epsilon$ and $M \geq N$. Then we have

$$
\begin{aligned}
& \left|A_{n_{1}, \cdots, n_{N}}^{\hat{\mathcal{F}}_{\alpha, \beta} F}-A_{n_{1}, \cdots, n_{N}}^{F} \beta^{n_{1}+\cdots+n_{N}}\right| \\
= & \left|\int_{C(Q)} \hat{\mathcal{F}}_{\alpha, \beta} F(x) \Psi_{n_{1}, \cdots, n_{N}}(x) m_{Y}(d x)-A_{n_{1}, \cdots, n_{N}}^{F} \beta^{n_{1}+\cdots+n_{N}}\right| \\
\leq & \left|\int_{C(Q)}\left[\hat{\mathcal{F}}_{\alpha, \beta} F(x)-\mathcal{F}_{\alpha, \beta} F_{M}(x)\right] \Psi_{n_{1}, \cdots, n_{N}}(x) m_{Y}(d x)\right| \\
& +\left|\int_{C(Q)} \mathcal{F}_{\alpha, \beta} F_{M}(x) \Psi_{n_{1}, \cdots, n_{N}}(x) m_{Y}(d x)-A_{n_{1}, \cdots, n_{N}}^{F} \beta^{n_{1}+\cdots+n_{N}}\right| .
\end{aligned}
$$

But by the Hölder inequality and the fact that $\left\{\Psi_{n_{1}, \cdots, n_{N}}\right\}$ is an orthonormal set,

$$
\left|\int_{C(Q)}\left[\hat{\mathcal{F}}_{\alpha, \beta} F(x)-\mathcal{F}_{\alpha, \beta} F_{M}(x)\right] \Psi_{n_{1}, \cdots, n_{N}}(x) m_{Y}(d x)\right| \leq\left\|\hat{\mathcal{F}}_{\alpha, \beta} F-\mathcal{F}_{\alpha, \beta} F_{M}\right\|_{2}<\epsilon
$$

and from (3.2) we know that

$$
\int_{C(Q)} \mathcal{F}_{\alpha, \beta} F_{M}(x) \Psi_{n_{1}, \cdots, n_{N}}(x) m_{Y}(d x)=A_{n_{1}, \cdots, n_{N}}^{F} \beta^{n_{1}+\cdots+n_{N}} .
$$

Hence

$$
\left|A_{n_{1}, \cdots, n_{N}}^{\hat{\mathcal{F}}_{\alpha, \beta} F}-A_{n_{1}, \cdots, n_{N}}^{F} \beta^{n_{1}+\cdots+n_{N}}\right|<\epsilon
$$

which establishes equation (3.7).

The following theorem is our main result. It gives a necessary and sufficient condition that a functional $F$ in $L_{2}(C(Q))$ has an integral transform $\hat{\mathcal{F}}_{\alpha, \beta} F$ belonging to $L_{2}(C(Q))$.

Theorem 3.5. Let $F \in L_{2}(C(Q))$ be given by (2.3) with Fourier-Hermite coefficients given by (2.4). Let $\alpha$ and $\beta$ be as in Lemma 2.1. Then $\hat{\mathcal{F}}_{\alpha, \beta} F$ exists and is an element of $L_{2}(C(Q))$ if and only if

$$
\lim _{N \rightarrow \infty} \sum_{n_{1}, \cdots, n_{N}=0}^{N}\left|A_{n_{1}, \cdots, n_{N}}^{F} \beta^{n_{1}+\cdots+n_{N}}\right|^{2}<\infty .
$$


Furthermore if (3.8) holds, then the Fourier-Hermite expression of $\hat{\mathcal{F}}_{\alpha, \beta} F$ is given by

$$
\hat{\mathcal{F}}_{\alpha, \beta} F(x)=\operatorname{li}_{N \rightarrow \infty} \text { i.m. } \sum_{n_{1}, \cdots, n_{N}=0}^{N} A_{n_{1}, \cdots, n_{N}}^{F} \beta^{n_{1}+\cdots+n_{N}} \Psi_{n_{1}, \cdots, n_{N}}(x)
$$

for $x \in C(Q)$.

Proof. Assume that $\hat{\mathcal{F}}_{\alpha, \beta} F$ exists and is an element of $L_{2}(C(Q))$. For any given $\epsilon>0$, we have $\left\|\hat{\mathcal{F}}_{\alpha, \beta} F-\mathcal{F}_{\alpha, \beta} F_{N}\right\|_{2}<\epsilon$ for sufficiently large $N$, and so

$$
\left(\sum_{n_{1}, \cdots, n_{N}=0}^{N}\left|A_{n_{1}, \cdots, n_{N}}^{F} \beta^{n_{1}+\cdots+n_{N}}\right|^{2}\right)^{1 / 2}=\left\|\mathcal{F}_{\alpha, \beta} F_{N}\right\|_{2} \leq\left\|\hat{\mathcal{F}}_{\alpha, \beta} F\right\|_{2}+\epsilon .
$$

Hence we have

$$
\lim _{N \rightarrow \infty} \sum_{n_{1}, \cdots, n_{N}=0}^{N}\left|A_{n_{1}, \cdots, n_{N}}^{F} \beta^{n_{1}+\cdots+n_{N}}\right|^{2} \leq\left\|\hat{\mathcal{F}}_{\alpha, \beta} F\right\|_{2}^{2}<\infty .
$$

To prove the converse, suppose that (3.8) holds. Let $M>N$, let

$$
I_{M}=\left\{\left(n_{1}, \cdots, n_{M}\right): n_{1}, \cdots, n_{M}=0,1, \cdots, M\right\},
$$

and let

$$
I_{N}=\left\{\left(n_{1}, \cdots, n_{M}\right): n_{1}, \cdots, n_{N}=0,1, \cdots, N \text { and } n_{N+1}=\cdots=n_{M}=0\right\} .
$$

Then

$$
\begin{aligned}
& \left\|\mathcal{F}_{\alpha, \beta} F_{M}-\mathcal{F}_{\alpha, \beta} F_{N}\right\|_{2}^{2} \\
= & \left\|\sum_{I_{M}-I_{N}} A_{n_{1}, \cdots, n_{M}}^{F} \beta^{n_{1}+\cdots+n_{M}} \Psi_{n_{1}, \cdots, n_{M}}\right\|_{2}^{2} \\
= & \sum_{I_{M}-I_{N}}\left|A_{n_{1}, \cdots, n_{M}}^{F} \beta^{n_{1}+\cdots+n_{M}}\right|^{2} \\
= & \sum_{n_{1}, \cdots, n_{M}=0}^{M}\left|A_{n_{1}, \cdots, n_{M}}^{F} \beta^{n_{1}+\cdots+n_{M}}\right|^{2}-\sum_{n_{1}, \cdots, n_{N}=0}^{N}\left|A_{n_{1}, \cdots, n_{N}}^{F} \beta^{n_{1}+\cdots+n_{N}}\right|^{2}
\end{aligned}
$$

which goes to 0 as $M, N \rightarrow \infty$. Hence $\left\{\mathcal{F}_{\alpha, \beta} F_{N}\right\}$ is a Cauchy sequence in $L_{2}(C(Q))$ and since $L_{2}(C(Q))$ is complete,

$$
\hat{\mathcal{F}}_{\alpha, \beta} F(x)=\underset{N \rightarrow \infty}{\lim . \operatorname{m}} \mathcal{F}_{\alpha, \beta} F_{N}(x), \quad x \in C(Q)
$$

exists and is an element of $L_{2}(C(Q))$ and is given by (3.9). 
Our first corollary follows immediately from Theorem 3.5.

Corollary 3.6. Let $F, \alpha$ and $\beta$ be as in Theorem 3.5. Furthermore assume that $|\beta| \leq 1$. Then $\hat{\mathcal{F}}_{\alpha, \beta} F$ exists, belongs to $L_{2}(C(Q))$, and

$$
\begin{aligned}
\left\|\hat{\mathcal{F}}_{\alpha, \beta} F\right\|_{2}^{2} & =\lim _{N \rightarrow \infty} \sum_{n_{1}, \cdots, n_{N}=0}^{N}\left|A_{n_{1}, \cdots, n_{N}}^{F} \beta^{n_{1}+\cdots+n_{N}}\right|^{2} \\
& \leq \lim _{N \rightarrow \infty} \sum_{n_{1}, \cdots, n_{N}=0}^{N}\left|A_{n_{1}, \cdots, n_{N}}^{F}\right|^{2}=\|F\|_{2}^{2} .
\end{aligned}
$$

In addition,

$$
\left\|\hat{\mathcal{F}}_{\alpha, \beta} F\right\|_{2}=\|F\|_{2}
$$

if and only if $|\beta|=1$.

The following corollary is immediate from Theorems 3.3 and 3.5.

Corollary 3.7. Let $F, \alpha$ and $\beta$ be as in Theorem 3.5 and let $c$ be a nonzero real number. Then

$$
\hat{\mathcal{F}}_{\alpha, c \beta} F(x)=\lim _{N \rightarrow \infty} \sum_{n_{1}, \cdots, n_{N}=0}^{N} A_{n_{1}, \cdots, n_{N}}^{F} \beta^{n_{1}+\cdots+n_{N}} \Psi_{n_{1}, \cdots, n_{N}}(c x)
$$

for $x \in C(Q)$.

Next choosing $\alpha=\sqrt{2}$ and $\beta=i$, we obtain a Yeh-Wiener space version of the main theorem of [4].

Corollary 3.8. Every functional $F(x) \in L_{2}(C(Q))$ has a Fourier-Wiener transform $G(x) \in L_{2}(C(Q))$. The functional $G(x)$ has $F(-x)$ as its transform and $F$ and $G$ satisfies Plancherel's relation

$$
\int_{C(Q)}|F(x)|^{2} m_{Y}(d x)=\int_{C(Q)}|G(x)|^{2} m_{Y}(d x) .
$$

Proof. Using Corollary 3.6 and Theorem 3.5, we obtain that $G(x) \in L_{2}(C(Q))$ is given by

$$
G(x)=\underset{N \rightarrow \infty}{\operatorname{li} . \operatorname{m}} \sum_{n_{1}, \cdots, n_{N}=0}^{N} A_{n_{1}, \cdots, n_{N}}^{F} i^{n_{1}+\cdots+n_{N}} \Psi_{n_{1}, \cdots, n_{N}}(x),
$$

and that

$$
\hat{\mathcal{F}}_{\sqrt{2}, i} G(x)=\lim _{N \rightarrow \infty} \sum_{n_{1}, \cdots, n_{N}=0}^{N} A_{n_{1}, \cdots, n_{N}}^{F}(-1)^{n_{1}+\cdots+n_{N}} \Psi_{n_{1}, \cdots, n_{N}}(x) .
$$


But since the Hermite polynomial $H_{n}$ is an even function if $n$ is even and an odd function if $n$ is odd, it is easy to see that

$$
(-1)^{n_{1}+\cdots+n_{N}} \Psi_{n_{1}, \cdots, n_{N}}(x)=\Psi_{n_{1}, \cdots, n_{N}}(-x)
$$

and so $\hat{\mathcal{F}}_{\sqrt{2}, i} G(x)=F(-x)$. Finally equation (3.13) follows immediately from (3.11).

Recall that throughout this paper we have assumed that $\beta=a+b i$ was a nonzero complex number satisfying inequality (2.5); namely that $\operatorname{Re}\left(1-\beta^{2}\right)>$ 0. Furthermore, in Corollary 3.6 we showed that if $\beta$ also satisfies the inequality $|\beta| \leq 1$, then $\hat{\mathcal{F}}_{\alpha, \beta} F$ exists as an element of $L_{2}(C(Q))$ for all $F \in L_{2}(C(Q))$ with $\alpha$ given by (2.6). In Example 10 of [11], Kim and Skoug showed that for any complex number $\beta$ with $|\beta|>1$ and $\operatorname{Re}\left(1-\beta^{2}\right)>0$, there exists a functional $F \in L_{2}\left(C_{0}[0, T]\right)$ such that $\mathcal{F}_{\alpha, \beta} F, \hat{\mathcal{F}}_{\alpha, \beta} F$ in our notation, doesn't exist as an element of $L_{2}\left(C_{0}[0, T]\right)$. Using the same idea as in Example 10 of [11], we can construct a functional $F \in L_{2}(C(Q))$ such that $\hat{\mathcal{F}}_{\alpha, \beta} F$ doesn't exist as an element of $L_{2}(C(Q))$ when $\beta$ is a complex number with $|\beta|>1$ and $\operatorname{Re}\left(1-\beta^{2}\right)>0$.

Our final results involves the inverse transform of $\hat{\mathcal{F}}_{\alpha, \beta}$. In order to insure the existence of the inverse transform of $\hat{\mathcal{F}}_{\alpha, \beta}$ we need to put an additional assumption on $\beta=a+b i$; namely that

$$
\operatorname{Re}\left(1-\frac{1}{\beta^{2}}\right)>0 .
$$

Now $\operatorname{Re}\left(1-1 / \beta^{2}\right)>0$ if and only if $\left(a^{2}+b^{2}\right)^{2}-\left(a^{2}-b^{2}\right)>0$. But the graph of $\left(a^{2}+b^{2}\right)^{2}-\left(a^{2}-b^{2}\right)=0$ is the lemniscate $r^{2}=\cos (2 \theta)$. Hence $\operatorname{Re}\left(1-1 / \beta^{2}\right)>0$ if and only if the point $(a, b) \in \mathbb{R}^{2}$ lies outside the lemniscate $\left(a^{2}+b^{2}\right)^{2}-\left(a^{2}-b^{2}\right)=0$.

Theorem 3.9. Let $F, \alpha$ and $\beta$ be as in Theorem 3.5 and assume that (3.8) holds. Furthermore assume that $\beta$ satisfies inequality (3.14). Then for $\alpha^{\prime} \equiv \sqrt{1-1 / \beta^{2}}$ and $\beta^{\prime}= \pm 1 / \beta$, we have that

$$
\hat{\mathcal{F}}_{\alpha^{\prime}, \beta^{\prime}} \hat{\mathcal{F}}_{\alpha, \beta} F(x)=F\left(\beta \beta^{\prime} x\right), \quad x \in C(Q) .
$$

That is to say,

$$
\hat{\mathcal{F}}_{\alpha^{\prime}, 1 / \beta} \hat{\mathcal{F}}_{\alpha, \beta} F(x)=F(x), \quad x \in C(Q)
$$

and

$$
\hat{\mathcal{F}}_{\alpha^{\prime},-1 / \beta} \hat{\mathcal{F}}_{\alpha, \beta} F(x)=F(-x), \quad x \in C(Q) .
$$

Proof. Since $\hat{\mathcal{F}}_{\alpha, \beta} F$ exists, the Fourier-Hermite expression of it is given by

$$
\hat{\mathcal{F}}_{\alpha, \beta} F(x)=\underset{N \rightarrow \infty}{\operatorname{in} .} \sum_{n_{1}, \cdots, n_{N}=0}^{N} A_{n_{1}, \cdots, n_{N}}^{F} \beta^{n_{1}+\cdots+n_{N}} \Psi_{n_{1}, \cdots, n_{N}}(x)
$$


for $x \in C(Q)$. Now since $\beta \beta^{\prime}= \pm 1$, we have

$$
\begin{aligned}
\lim _{N \rightarrow \infty} \sum_{n_{1}, \cdots, n_{N}=0}^{N}\left|A_{n_{1}, \cdots, n_{N}}^{F} \beta^{n_{1}+\cdots+n_{N}}\left(\beta^{\prime}\right)^{n_{1}+\cdots+n_{N}}\right|^{2} & =\lim _{N \rightarrow \infty} \sum_{n_{1}, \cdots, n_{N}=0}^{N}\left|A_{n_{1}, \cdots, n_{N}}^{F}\right|^{2} \\
& =\|F\|_{2}^{2}<\infty .
\end{aligned}
$$

Hence by Theorem $3.5, \hat{\mathcal{F}}_{\alpha^{\prime}, \beta^{\prime}} \hat{\mathcal{F}}_{\alpha, \beta} F$ exists and is given by

$$
\begin{aligned}
\hat{\mathcal{F}}_{\alpha^{\prime}, \beta^{\prime}} \hat{\mathcal{F}}_{\alpha, \beta} F(x) & =\lim _{\dot{N} \rightarrow \infty} \sum_{n_{1}, \cdots, n_{N}=0}^{N} A_{n_{1}, \cdots, n_{N}}^{F}\left(\beta \beta^{\prime}\right)^{n_{1}+\cdots+n_{N}} \Psi_{n_{1}, \cdots, n_{N}}(x) \\
& =\lim _{N \rightarrow \infty} \text { i.m. } \sum_{n_{1}, \cdots, n_{N}=0}^{N} A_{n_{1}, \cdots, n_{N}}^{F} \Psi_{n_{1}, \cdots, n_{N}}\left(\beta \beta^{\prime} x\right) \\
& =F\left(\beta \beta^{\prime} x\right),
\end{aligned}
$$

for $x \in C(Q)$, where the second equality holds since $\beta \beta^{\prime}=1$ or -1 , and this completes the proof of Theorem 3.9.

The following corollary is immediate from Theorems 3.3 and 3.9 .

Corollary 3.10. Let $F, \alpha, \beta, \alpha^{\prime}$ and $\beta^{\prime}$ be as in Theorem 3.9. Let $c$ and $c^{\prime}$ be nonzero real numbers. Then

$$
\hat{\mathcal{F}}_{\alpha^{\prime}, c^{\prime} \beta^{\prime}} \hat{\mathcal{F}}_{\alpha, c \beta} F(x)=F\left(c c^{\prime} \beta \beta^{\prime} x\right)
$$

for $x \in C(Q)$.

\section{References}

[1] E. Berkson and T. A. Gillespie, Absolutely continuous functions of two variables and well-bounded operators, J. London Math. Soc., 30(1984), 305-321.

[2] R. H. Cameron, Some examples of Fourier-Wiener transforms of analytic functionals, Duke Math. J., 12(1945), 485-488.

[3] R. H. Cameron and W. T. Martin, Fourier-Wiener transforms of analytic functionals, Duke Math. J., 12(1945), 489-507.

[4] R. H. Cameron and W. T. Martin, Fourier-Wiener transforms of functionals belonging to $L_{2}$ over the space C, Duke Math. J., 14(1947), 99-107.

[5] R. H. Cameron and D. A. Storvick, An $L_{2}$ analytic Fourier-Feynman transform, Michigan Math. J., 23(1976), 1-30.

[6] K. S. Chang, B. S. Kim and I. Yoo, Integral transform and convolution of analytic functionals on abstract Wiener spaces, Numer. Funct. Anal. Optim., 21(2000), 97-105. 
[7] E. W. Hobson, The theory of functions of a real variable and the theory of Fourier's series, Vol. 1 (3rd ed.), Dover, New York, 1957.

[8] G. W. Johnson and D. L. Skoug, A stochastic integration formula for two-parameter Wiener $\times$ two-parameter Wiener space, SIAM J. Math. Anal., 18(1987), 919-932.

[9] B. J. Kim, B. S. Kim and D. Skoug, Integral transforms, convolution products, and first variations, Internat. J. Math. Math. Sci., 2004(2004), 579-598.

[10] B. J. Kim, B. S. Kim and I. Yoo, Integral transforms of functionals on a function space of two variables, submitted.

[11] B. S. Kim and D. Skoug, Integral transforms of functionals in $L_{2}\left(C_{0}[0, T]\right)$, Rocky Mountain J. Math., 33(2003), 1379-1393.

[12] Y. J. Lee, Integral transforms of analytic functions on abstract Wiener spaces, J. Funct. Anal., 47(1982), 153-164.

[13] D. Skoug and D. Storvick, A survey of results involving transforms and convolutions in function space, Rocky Mountain J. Math., 34(2004), 1147-1176.

[14] J. Yeh, Wiener measure in a space of functions of two variables, Trans. Amer. Math. Soc., 95(1960), 433-450.

[15] J. Yeh, Orthogonal developments of functionals and related theroems in the Wiener space of functions of two variables, Pacific J. Math., 13(1963), 14271436.

[16] J. Yeh, Stochastic processes and the Wiener integral, Marcel Dekker, New York, 1983. 